\title{
Global analysis of human duplicated genes reveals the relative importance of whole-genome duplicates originated in the early vertebrate evolution
}

\author{
Debarun Acharya and Tapash C. Ghosh ${ }^{*}$
}

\begin{abstract}
Background: Gene duplication is a genetic mutation that creates functionally redundant gene copies that are initially relieved from selective pressures and may adapt themselves to new functions with time. The levels of gene duplication may vary from small-scale duplication (SSD) to whole genome duplication (WGD). Studies with yeast revealed ample differences between these duplicates: Yeast WGD pairs were functionally more similar, less divergent in subcellular localization and contained a lesser proportion of essential genes. In this study, we explored the differences in evolutionary genomic properties of human SSD and WGD genes, with the identifiable human duplicates coming from the two rounds of whole genome duplication occurred early in vertebrate evolution.
\end{abstract}

Results: We observed that these two groups of duplicates were also dissimilar in terms of their evolutionary and genomic properties. But interestingly, this is not like the same observed in yeast. The human WGDs were found to be functionally less similar, diverge more in subcellular level and contain a higher proportion of essential genes than the SSDs, all of which are opposite from yeast. Additionally, we explored that human WGDs were more divergent in their gene expression profile, have higher multifunctionality and are more often associated with disease, and are evolutionarily more conserved than human SSDs.

Conclusions: Our study suggests that human WGD duplicates are more divergent and entails the adaptation of WGDs to novel and important functions that consequently lead to their evolutionary conservation in the course of evolution.

Keywords: Small-scale duplication, Whole-genome duplication, Functional divergence, Gene essentiality, Disease genes, Protein multifunctionality, Evolutionary rate

\section{Background}

Gene duplication is a key source for generating new gene copies from pre-existing ones [1-3]. These newly-made gene copies are initially functionally redundant and relieved from selective pressure, and may adapt themselves to new functions $[2,4-6]$. Thus, many of the previous studies concluded gene duplication as the primary guiding force of organism evolution for providing raw genetic materials for genome evolution [1, 2, 7]. Although, the retention of

\footnotetext{
* Correspondence: tapash@jcbose.ac.in

Bioinformatics Centre, Bose Institute, P 1/12, C.I.T. Scheme VII M, Kolkata 700054West Bengal, India
}

duplicated genes is not a trouble-free process and most of the duplicates become nonfunctionalized and/ or lost from the genome [2], whereas others become fixed within the genome in course of evolution. The retention of duplicates might be initially favourable due to circumstances like increased gene dosage advantage, where the duplication and subsequent increase in the gene product may be advantageous to the organism [5, 8]. Additionally, gene duplicates may serve as backup copies capable of functional compensation upon gene deletion [9] and provide increased genetic robustness against deleterious mutations [10], but their maintenance requires stringent regulation in 
gene dosage [11, 12] or expression patterns [13-16]. That apart, the duplicates may either diverge at the subcellular protein localization [17] or share the ancestral function [18] after complementary degenerative mutations (subfunctionalization) [19] or adapt to new functions (neofunctionalization) [2]. Furthermore, there are also subtle differences in the extent of gene duplication. In most of the cases, duplication involves a single gene and termed as small-scale duplication (SSD), whereas, large-scale duplications may involve many genes, chromosomal segments or even the entire genome, with the latter being known as wholegenome duplication (WGD) [20]. Although small-scale duplication can occur at any time and may be retained in course of evolution, there are a few evidences of whole genome duplication in eukaryotic organisms, being most common and widely studied in the evolution of plant genome [21-24]. Many previous studies highlighted the evidence of an ancient WGD in the yeast genome [25-27]. Additionally, evidence of two rounds of whole-genome duplication was also prominent in the early vertebrate evolution [28-33], which provides the raw materials for increasing genome and organism complexity and extensive species diversity $[29,31]$ and hence, is an important process in vertebrate evolution [30,31].

However, as genes' functions are mainly mediated by their encoded proteins, which primarily function with the association of other such proteins [34], the proper functioning of a gene depends on the stoichiometric balance of the proteins participants. The retention of duplicated genes creates a stoichiometric disparity in the protein-protein interaction network, with the duplicated genes producing more proteins than the non-duplicated ones [35-37]. The two extent of duplication affect their associated protein-interaction network differentially [20,38-41]. In WGD, the whole PPI network becomes simultaneously duplicated, and the stoichiometric balance of the participant proteins remains the same; whereas in SSD, the duplicated gene tends to form more protein in contrast to the nonduplicated interacting partners, thereby creating an imbalance in the whole PPI network. Therefore, in general, whole-genome duplicates are expected to be retained intact within the genome [39].

Most of the studies highlighting gene duplication compared the attributes of duplicated genes with that of singletons $[10,42,43]$. This raised an important question-are all duplicates equal in their genomic and evolutionary characteristics? With the well-established gene duplication data in yeast, it became possible to identify the duplicates originated from whole-genome duplication as well as those from small-scale duplication [25]. Comparing these two distinct duplicate groups, researchers observed quantifiable differences in yeast [20, 41, 44]. They found that the yeast WGDs are functionally more similar than SSD genes, which is independent of their sequence similarity $[20,44]$. Additionally, yeast SSDs also diverge more at their subcellular localization than the WGDs [41]. Also, yeast SSD genes were found to contain a higher proportion of essential genes than WGD genes [20,44].

The occurrence of two rounds of whole-genome duplication in early vertebrate lineage [28-33] and the subsequent detection of traces of these whole-genome duplicates in human $[32,39,45]$ lead us to differentiate the genomic and evolutionary attributes of human small-scale and whole-genome duplicates. As the human WGDs stem from the ancient two rounds of genome duplication that had occurred in early vertebrates, it can be stated that these human duplicates became subjected to more evolutionary pressure due to their long term evolutionary exposure than that in yeast. Therefore, our study will explore the relative importance and the long-term fate of these whole-genome duplicates that had originated during the early vertebrate evolution in contrast to the duplicates originating spontaneously at small-scale.

\section{Results}

\section{Functional similarity of human SSD and WGD genes}

The functional similarities between each pair of human small-scale and whole-genome duplicates were calculated using the Gene Ontology (GO) annotation from the biomart interface of Ensembl (version 77) [46], using GO domains 'biological process' as well as 'molecular function'. We obtained a higher functional similarity in small-scale duplicates than the whole-genome duplicated group (Table 1). However, the functional diversification of paralogs is dependent on their nonsynonymous nucleotide substitution per nonsynonymous site $(\mathrm{dN})$, and the whole-genome duplicates tend to have a higher $\mathrm{dN}$ value the small-scale duplicates, for being evolutionarily more ancient. Therefore, we binned our dataset according to different $\mathrm{dN}$ ranges (nonsynonymous nucleotide substitution per nonsynonymous site) (see Materials and methods) and compared the functional similarity between SSD and WGD duplicate pairs. This approach is similar to that adopted by Hakes et al. [20]. We found that SSD duplicate pairs are functionally more similar than the WGD pairs in each $\mathrm{dN}$ range (Table 1) considering both their involvement in biological processes and molecular function (Fig. 1). In other terms, human WGD pairs were found to be functionally more divergent, independent of their sequence divergence. 
Table 1 Differences between the properties of human small-scale and whole-genome duplicate pairs in different dN ranges. Pair wise two-tailed Mann-Whitney $U$ test were used to compare the means of SSD and WGD pairs within each group

\begin{tabular}{|c|c|c|c|c|c|c|c|c|c|c|}
\hline \multirow{2}{*}{$\begin{array}{l}\text { Parameter } \\
\text { Measured }\end{array}$} & \multirow[t]{2}{*}{ Database used } & \multicolumn{3}{|l|}{ Overall } & \multicolumn{3}{|c|}{$\mathrm{dN} 0.0-0.1$} & \multicolumn{3}{|c|}{$\mathrm{dN} 0.1-0.2$} \\
\hline & & SSD & WGD & P-value & SSD & WGD & P-value & SSD & WGD & P-value \\
\hline \multirow{4}{*}{$\begin{array}{l}\text { Functional Similarity } \\
\text { between paralogs }\end{array}$} & \multirow{2}{*}{$\begin{array}{l}\text { Shared GO Terms } \\
\text { for Biological } \\
\text { Process }\end{array}$} & $\bar{x}=0.710$ & $\bar{x}=0.415$ & \multirow[t]{2}{*}{$<1.00 \times 10^{-6}$} & $\bar{x}=0.734$ & $\bar{x}=0.499$ & \multirow[t]{2}{*}{$2.325 \times 10^{-52}$} & $\bar{x}=0.720$ & $\bar{x}=0.476$ & \multirow[t]{2}{*}{$5.925 \times 10^{-123}$} \\
\hline & & $N=14742$ & $N=12022$ & & $N=3640$ & $N=414$ & & $N=2754$ & $N=1140$ & \\
\hline & \multirow{2}{*}{$\begin{array}{l}\text { Shared GO Terms } \\
\text { for Molecular } \\
\text { Function }\end{array}$} & $\bar{x}=0.840$ & $\bar{x}=0.659$ & \multirow[t]{2}{*}{$<1.00 \times 10^{-6}$} & $\bar{x}=0.850$ & $\bar{x}=0.724$ & \multirow[t]{2}{*}{$6.077 \times 10^{-47}$} & $\bar{x}=0.856$ & $\bar{x}=0.706$ & \multirow[t]{2}{*}{$1.075 \times 10^{-129}$} \\
\hline & & $N=18584$ & $N=12392$ & & $N=4668$ & $N=410$ & & $N=3510$ & $N=1188$ & \\
\hline \multirow{2}{*}{$\begin{array}{l}\text { Shared Subcellular } \\
\text { Compartment } \\
\text { of paralogs }\end{array}$} & \multirow{2}{*}{$\begin{array}{l}\text { GO Cellular } \\
\text { Component }\end{array}$} & $\bar{x}=0.782$ & $\bar{x}=0.541$ & \multirow[t]{2}{*}{$<1.00 \times 10^{-6}$} & $\bar{x}=0.816$ & $\bar{x}=0.579$ & \multirow[t]{2}{*}{$5.341 \times 10^{-76}$} & $\bar{x}=0.788$ & $\bar{x}=0.581$ & \multirow[t]{2}{*}{$5.652 \times 10^{-119}$} \\
\hline & & $N=15248$ & $N=12198$ & & $N=3790$ & $N=380$ & & $N=2914$ & $N=1162$ & \\
\hline \multirow{4}{*}{$\begin{array}{l}\text { Gene expression } \\
\text { profile similarity } \\
\text { between paralogs }\end{array}$} & \multirow{2}{*}{$\begin{array}{l}\text { Human Protein } \\
\text { Atlas }\end{array}$} & $\bar{x}=0.403$ & $\bar{x}=0.193$ & \multirow[t]{2}{*}{$<1.00 \times 10^{-6}$} & $\bar{x}=0.615$ & $\bar{x}=0.254$ & \multirow[t]{2}{*}{$1.558 \times 10^{-63}$} & $\bar{x}=0.414$ & $\bar{x}=0.253$ & \multirow[t]{2}{*}{$1.774 \times 10^{-42}$} \\
\hline & & $N=11726$ & $N=13060$ & & $N=2588$ & $N=426$ & & $N=2758$ & $N=1226$ & \\
\hline & \multirow{2}{*}{$\begin{array}{l}\text { Expression } \\
\text { Atlas }\end{array}$} & $\bar{x}=0.450$ & $\bar{x}=0.216$ & \multirow[t]{2}{*}{$<1.00 \times 10^{-6}$} & $\bar{x}=0.508$ & $\bar{x}=0.284$ & \multirow[t]{2}{*}{$1.032 \times 10^{-30}$} & $\bar{x}=0.457$ & $\bar{x}=0.280$ & \multirow[t]{2}{*}{$5.953 \times 10^{-53}$} \\
\hline & & $N=15404$ & $N=13072$ & & $N=3628$ & $N=422$ & & $N=3458$ & $N=1220$ & \\
\hline
\end{tabular}


Table 1 Differences between the properties of human small-scale and whole-genome duplicate pairs in different dN ranges. Pair wise two-tailed Mann-Whitney $U$ test were used to compare the means of SSD and WGD pairs within each group (Continued)

\begin{tabular}{|c|c|c|c|c|c|c|c|c|c|c|}
\hline \multirow{2}{*}{$\begin{array}{l}\text { Parameter } \\
\text { Measured }\end{array}$} & \multirow[t]{2}{*}{ Database used } & \multicolumn{3}{|c|}{$\mathrm{dN} 0.2-0.3$} & \multicolumn{3}{|c|}{ dN 0.3-0.4 } & \multicolumn{3}{|l|}{$\mathrm{dN}>0.4$} \\
\hline & & SSD & WGD & P-value & SSD & WGD & P-value & SSD & WGD & P-value \\
\hline \multirow{4}{*}{$\begin{array}{l}\text { Functional Similarity } \\
\text { between paralogs }\end{array}$} & \multirow{2}{*}{$\begin{array}{l}\text { Shared GO Terms } \\
\text { for Biological } \\
\text { Process }\end{array}$} & $\bar{x}=0.657$ & $\bar{x}=0.440$ & $2.892 \times 10^{-120}$ & $\bar{x}=0.726$ & $\bar{x}=0.413$ & $1.397 \times 10^{-274}$ & $\bar{x}=0.710$ & $\bar{x}=0.391$ & $1.983 \times 10^{-135}$ \\
\hline & & $N=3328$ & $N=2002$ & & $N=4264$ & $N=2192$ & & $N=756$ & $N=6274$ & \\
\hline & \multirow{2}{*}{$\begin{array}{l}\text { Shared GO Terms } \\
\text { for Molecular } \\
\text { Function }\end{array}$} & $\bar{x}=0.810$ & $\bar{x}=0.696$ & $1.814 \times 10^{-96}$ & $\bar{x}=0.846$ & $\bar{x}=0.677$ & $4.976 \times 10^{-218}$ & $\bar{x}=0.826$ & $\bar{x}=0.628$ & $5.072 \times 10^{-107}$ \\
\hline & & $N=4300$ & $N=2076$ & & $N=5246$ & $N=2250$ & & $N=860$ & $N=6468$ & \\
\hline \multirow{2}{*}{$\begin{array}{l}\text { Shared Subcellular } \\
\text { Compartment } \\
\text { of paralogs }\end{array}$} & \multirow{2}{*}{$\begin{array}{l}\text { GO Cellular } \\
\text { Component }\end{array}$} & $\bar{x}=0.740$ & $\bar{x}=0.541$ & $3.344 \times 10^{-139}$ & $\bar{x}=0.781$ & $\bar{x}=0.555$ & $1.421 \times 10^{-215}$ & $\bar{x}=0.777$ & $\bar{x}=0.527$ & $1.156 \times 10^{-100}$ \\
\hline & & $N=3444$ & $N=2036$ & & $N=4356$ & $N=2228$ & & $N=744$ & $N=6392$ & \\
\hline \multirow{4}{*}{$\begin{array}{l}\text { Gene expression } \\
\text { profile similarity } \\
\text { between paralogs }\end{array}$} & \multirow{2}{*}{$\begin{array}{l}\text { Human Protein } \\
\text { Atlas }\end{array}$} & $\bar{x}=0.307$ & $\bar{x}=0.191$ & $7.331 \times 10^{-32}$ & $\bar{x}=0.316$ & $\bar{x}=0.190$ & $1.131 \times 10^{-34}$ & $\bar{x}=0.322$ & $\bar{x}=0.179$ & $1.308 \times 10^{-17}$ \\
\hline & & $N=2834$ & $N=2158$ & & $N=3042$ & $N=2366$ & & $N=504$ & $N=6884$ & \\
\hline & \multirow{2}{*}{$\begin{array}{l}\text { Expression } \\
\text { Atlas }\end{array}$} & $\bar{x}=0.430$ & $\bar{x}=0.216$ & $1.377 \times 10^{-105}$ & $\bar{x}=0.420$ & $\bar{x}=0.219$ & $5.471 \times 10^{-96}$ & $\bar{x}=0.394$ & $\bar{x}=0.199$ & $5.735 \times 10^{-36}$ \\
\hline & & $N=3792$ & $N=2166$ & & $N=3922$ & $N=2370$ & & $N=604$ & $N=6894$ & \\
\hline
\end{tabular}

\section{Subcellular localization of SSD and WGD pairs}

In addition to the functional divergence, insight into the function of a gene is associated with the location of its encoded protein within the cell at the subcellular level. Many previous studies reported that gene duplication and the functional redundancy of duplicates can often be neutralized at the protein level by the subcellular protein compartmentalization $[17,47,48]$. Therefore, we also considered the subcellular localization of their encoded proteins as an alternative and/or associated mechanism beside functional divergence of the duplicated genes. The localization of the protein can be obtained by using the Gene Ontology (GO) terms under the GO domain 'Cellular Component' against its gene identifier. The shared cellular component between the paralogous copies of all SSD and WGD genes were calculated (see Materials and methods). We observed an overall higher subcellular compartment sharing of SSD pairs than that of WGD pairs (Table 1). When we binned our dataset according to different $\mathrm{dN}$ ranges as mentioned previously, the trend remains the same for each $\mathrm{dN}$ range (Table 1, Fig. 2), which indicates that the SSD genes are more often colocalized, and WGD genes are significantly more diverged in their subcellular localization, irrespective of their sequence divergence.

Gene expression correlation between SSD and WGD pairs The divergence of duplicated genes and can also occur at the gene expression levels. Earlier studies suggested that the gene expression patterns of duplicated pairs often undergo a spatial variation [reviewed in $\mathrm{Li}$ et al. [15]], and this can be considered as a mechanism for their stable maintenance [13]. Therefore, it is essential to understand the co-expression of the paralogs in different tissues after gene duplication, which is measured using the gene expression profiles of the paralogous copies in a wide range of normal tissues [14-16]. We used the high-throughput recent RNA-seq gene expression data of a wide range of normal human tissues from the Human Protein Atlas [49] and Expression Atlas [50] (see Materials and methods for more details). However, we observed that human SSD pairs have higher expression profile similarity than the WGD counterparts as a whole, and in each $d N$ range (Table 1, Fig. 3), suggesting that the functionally redundant human SSD genes also have more correlated expression profiles, and WGDs tend to diverge more in gene expression patterns.

\section{Evolutionary rate of human SSD and WGD genes}

The differences of human SSD and WGD pairs in their evolutionary genomic attributes clearly suggest that the human WGDs may tend to adapt themselves to new functions and locations. To investigate this, we used the one-to-one Mouse as well as Chimpanzee orthologs (see Materials and methods for details) to compare the evolutionary rates of human SSD and WGD genes by the Nonsynonymous nucleotide substitution per nonsynonymous sites $(\mathrm{dN})$ and the $\frac{d N}{d S}$ ratio, where 'dS' denotes synonymous nucleotide substitution per synonymous sites. We obtained a significantly slower evolutionary rate in WGD genes than the SSD genes for both the cases (Table 2, Fig. 4), indicating that the human WGD genes are evolutionarily more conserved, besides being functionally more diverged than the SSD genes, which is also supported by a previous study [51] and is consistent with the idea of the slower evolutionary rate of duplicated genes following their adaptation to new circumstances as described in Jordan et al.[43]. 

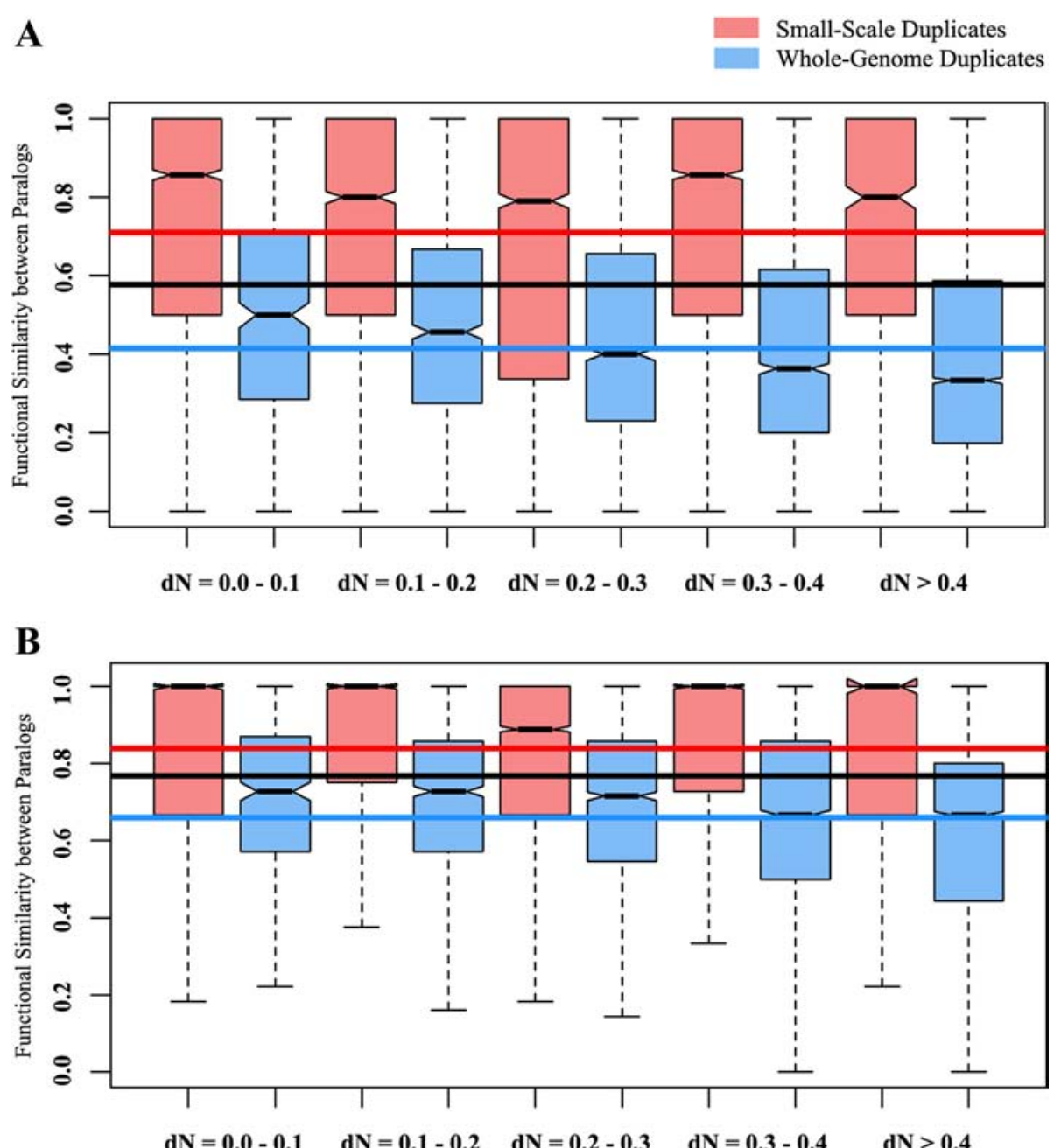

Fig. 1 Functional similarity between human small-scale and whole-genome duplicate pairs. The SSDs are represented in brick red and WGDs are represented in blue. The red and blue lines represent the mean functional similarity of SSD and WGD pairs, respectively. The black line represents the mean functional similarity of all human duplicates. The functional similarities between different $\mathrm{dN}$ ranges were calculated using both $\mathrm{GO}$ domains. a. Biological Process and $\mathbf{b}$. Molecular Function (For every dN range, $P<0.05$ ). For exact P-values, refer Table 1

\section{Multifunctionality of human SSD and WGD genes}

The higher probability of functional, sub-cellular localization and gene expression divergence of human WGD genes and their evolutionary conservation suggests that they may be associated with miscellaneous functions in contrast to the SSD counterparts. As our study is based on the functional fates of SSD and WGD genes, we were interested to observe which group is associated with more numerous functions. We used the unique GO biological process terms $[52,53]$ and the Pfam domain count [54] as proxies of multifunctionality (see Materials and methods). We observed that WGDonly genes are associated with more numerous Gene Ontology terms [Mean number of unique GO terms in $\mathrm{SSD} \approx 5$, Mean number of unique GO terms in WGD $\approx 10$, $P=6.707 \times 10^{-162}$, Mann Whitney $U$ test, $\mathrm{N}_{\mathrm{SSD}}=2569$, $\mathrm{N}_{\mathrm{WGD}}=5437$ ] [Fig. 5a] and contain significantly more domains in their encoded proteins [Mean number of Pfam domains in SSD $=1.61$, Mean number of Pfam domains in WGD $=2.02, P=1.130 \times 10^{-46}$, Mann Whitney $U$ test, $\mathrm{N}_{\mathrm{SSD}}=3060, \mathrm{~N}_{\mathrm{WGD}}=5607$ ] [Fig. 5b] than SSD-only genes. This suggests that human whole-genome duplicates are associated with more variety of functions than human SSD genes.

\section{Gene essentiality between human SSD and WGD genes}

So far, the comparison between the human SSD and the WGD genes showed that the SSD genes tend to diverge less in their function, subcellular localization, as well as in gene expression levels in different tissues. Additionally, WGD genes were also found to be evolutionarily more conserved and were adapted to new functions. But the importance of such functions from organismal perspective also plays a crucial role to get the whole 


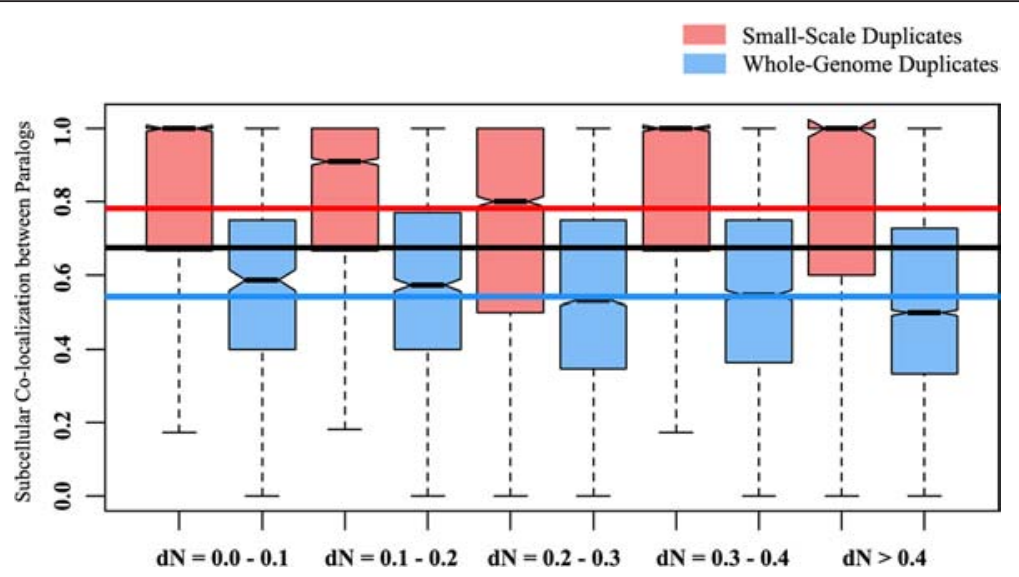

Fig. 2 Subcellular co-localization between human small-scale and whole-genome duplicate pairs. The SSDs are represented in brick red and WGDs are represented in blue. The red and blue lines represent the mean functional similarity of SSD and WGD pairs, respectively. The black line represents the mean functional similarity of all human duplicates (For every $\mathrm{dN}$ range, $P<0.05$ ). For exact $P$-values, see Table 1

picture. The importance of a gene can be measured in terms of gene essentiality. We used the Online GEne Essentiality (OGEE) Database [55] to obtain human essential genes [56] and observed a significantly higher proportion of essential genes among the WGD genes [Proportion of essential genes in SSD genes $=4.601 \%$, $\mathrm{N}_{\mathrm{SSD}}=2692$; Proportion of essential genes in WGD genes $\left.=11.344 \%, \mathrm{~N}_{\mathrm{WGD}}=5730\right][\mathrm{Z}=-9.99$, confidence level $99 \%$; $P<1.00 \times 10^{-4}$, two sample Z-test]. In other words, a greater portion of WGD genes shows lethality or sterility upon deletion than SSD genes, due to the absence of redundant paralogs in the former group.

\section{Disease association of human SSD and WGD genes}

Like gene essentiality, another important factor contributing to the importance of a gene in the organism is its disease association. It was previously hypothesised that gene duplication creates additional gene copies, and the increased functional redundancy can reduce the probability of disease formation by functional restoration upon gene deletion [57-59]. Therefore, the disease genes should remain as singletons [60]. More recently, studies linking gene duplication with disease hypothesise that duplication increase genetic redundancy, which in turn prefers accumulation of disease-associated mutations on the duplicates and hence, the duplicates may be more disease prone than the singletons [61]. Works with Mendelian disease genes reported their association with WGD genes [39, 62]. For our study, we considered all human disease associated genes from the Human Gene Mutation Database (HGMD) [63], which contains both Mendelian (monogenic) and complex (polygenic) disease genes. We observed that the proportion of disease genes is much higher among genes originating from whole-genome duplication [Proportion of disease genes in WGD genes $\left.=61.46 \%, \mathrm{~N}_{\mathrm{WGD}}=5908\right]$; than the small-scale duplicates [Proportion of disease genes in
SSD genes $\left.=27.89 \%, \mathrm{~N}_{\mathrm{SSD}}=3478\right][\mathrm{Z}=-31.420$, confidence level $99 \% ; P<1.00 \times 10^{-4}$, two sample Z-test]. This suggests that the reduction of functional redundancy in WGD genes increases disease susceptibility, and the increased ability of functional restoration reduce disease association of SSD genes.

\section{Discussions}

Gene duplication is the major source of genetic novelty that brings about genomic evolution. The term 'genetic novelty' comprise the generation of new genes from the pre-existing ones by mutation. Genetic mutation creates structural changes within the DNA which may lead to changes in the protein structure as well as its function. Although initially the duplicates are functionally redundant, they may either diverge or be maintained as backup copies during the course of evolution [2, 7, 64]. Recent studies with yeast confirmed that the wholegenome duplication maintains the stoichiometry of protein interaction network by increasing the dosage of its every participant, and small-scale duplication creates a stoichiometric imbalance within the network and hence, become functionally more divergent to maintain this balance [20, 38-41]. However, with the increasing organismal complexity and the genetic robustness, the whole-genome duplicates may also adapt to new functions, besides maintaining the resilience of protein interaction network. It will therefore be very interesting to explore the long-term fates of whole-genome duplication by observing human whole-genome duplicates (WGD), as the identifiable WGDs in human are traced from long back in the evolutionary scale i.e. from the two rounds of whole-genome duplication that had occurred in early vertebrate evolution. Therefore they must be evolved during the course of evolution from early vertebrates (like fish) to humans. In this study, we explored the 


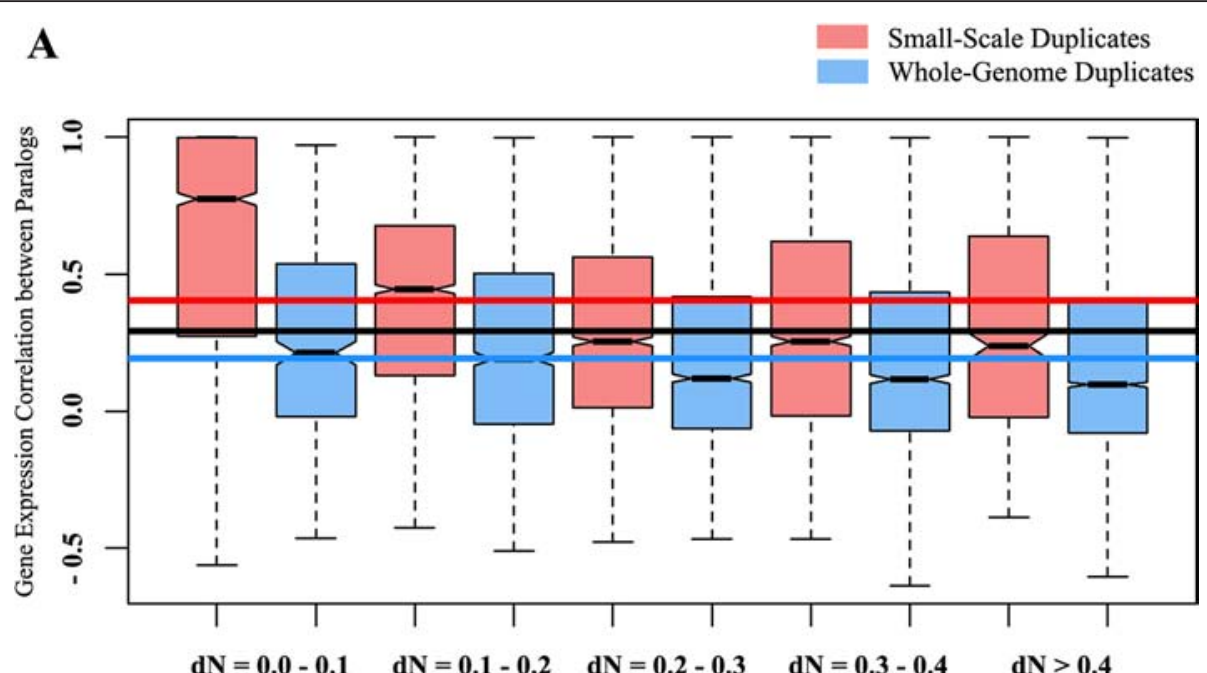

B

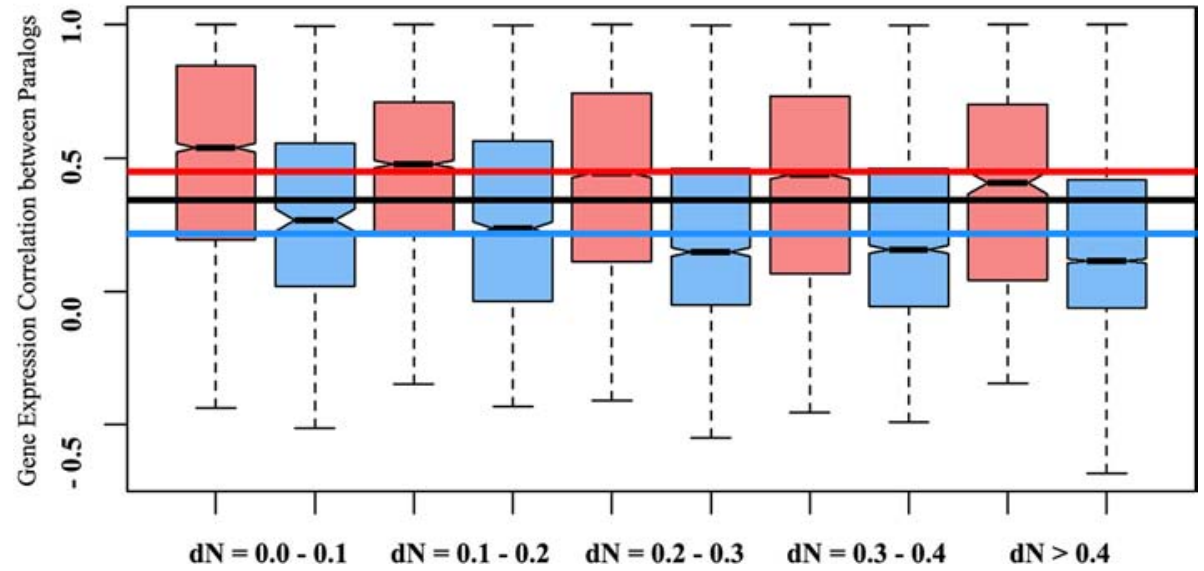

Fig. 3 Differences in gene expression correlation between human small-scale and whole-genome duplicate pairs. The gene expression correlation values of SSD and WGD pairs were calculated using RNA-seq gene expression data from $\mathbf{a}$. Human Protein Atlas and $\mathbf{b}$. Expression Atlas. The SSDs are represented in brick red and WGDs are represented in blue. The red and blue lines represent the mean gene expression correlation of SSD and WGD pairs, respectively. The black line represents the mean of gene expression correlation of all human duplicated gene pairs. (For every $\mathrm{dN}$ range, $P<0.05)$. Exact $P$-values are provided in Table 1

distinguishable differences between human small-scale and whole-genome duplicates. As we mentioned, the small-scale (SSD) and whole genome duplicates (WGD) are not similar in terms of their origin, and therefore in sequence divergence. So, we binned our datasets according to the non-synonymous nucleotide substitutions $(\mathrm{dN})$ to compare the similarities in evolutionary genomic properties between SSD and WGD duplicates independent of sequence changes that bring changes in amino acids, and in turn encoded proteins [20]. We observed that the human SSD and WGD duplicates were not similar in terms of their evolutionary and genomic properties. Based on their gene ontology terms, we found that WGD pairs share less functional similarity

Table 2 The evolutionary rate differences between human small-scale and whole-genome duplication using mouse (Mus musculus) and chimpanzee (Pan troglodytes) as outgroups. Two-tailed Mann-Whitney U-Test was used for comparisons between groups

\begin{tabular}{|c|c|c|c|c|c|c|}
\hline Outgroup Used & Gene Group & Number of genes & Mean dN & P-value & Mean $\frac{d N}{d S}$ & P-value \\
\hline \multirow[t]{2}{*}{ Mouse (Mus musculus) } & Human Small-Scale Duplicates & 958 & 0.089 & $6.212 \times 10^{-18}$ & 0.135 & $2.415 \times 10^{-12}$ \\
\hline & Human Whole-Genome Duplicates & 5689 & 0.062 & & 0.101 & \\
\hline \multirow[t]{2}{*}{ Chimpanzee (Pan troglodytes) } & Human Small-Scale Duplicates & 1611 & 0.012 & $3.842 \times 10^{-99}$ & 0.480 & $2.410 \times 10^{-76}$ \\
\hline & Human Whole-Genome Duplicates & 5309 & 0.006 & & 0.257 & \\
\hline
\end{tabular}


A

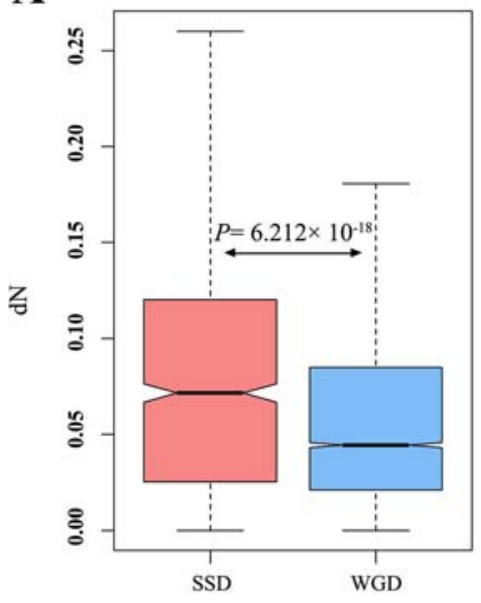

Using one-to-one Mouse orthologs

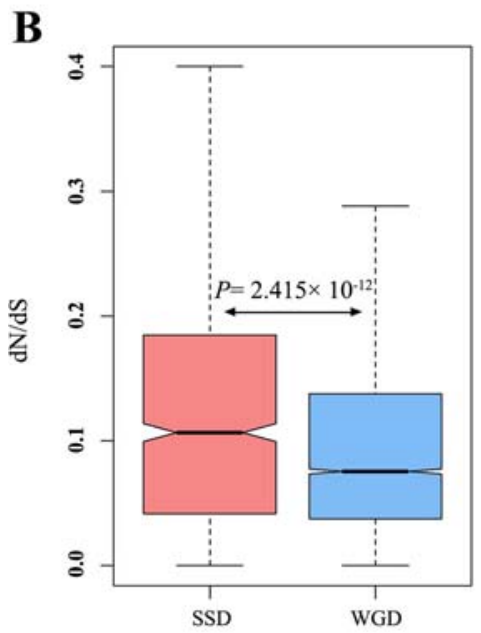

Using one-to-one Mouse orthologs
Small-Scale Duplicates

Whole-Genome Duplicates

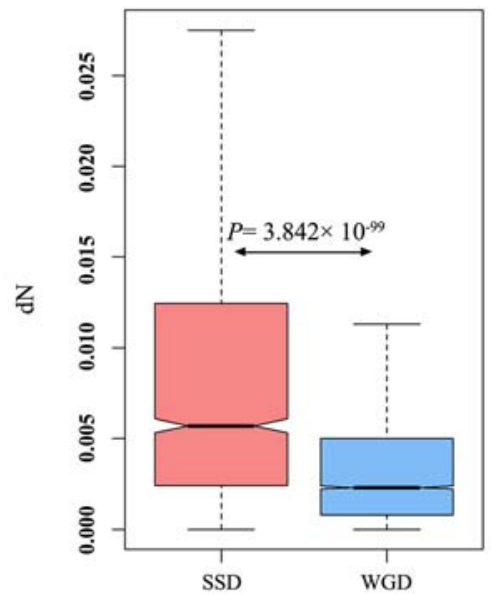

Using one-to-one Chimpanzee orthologs

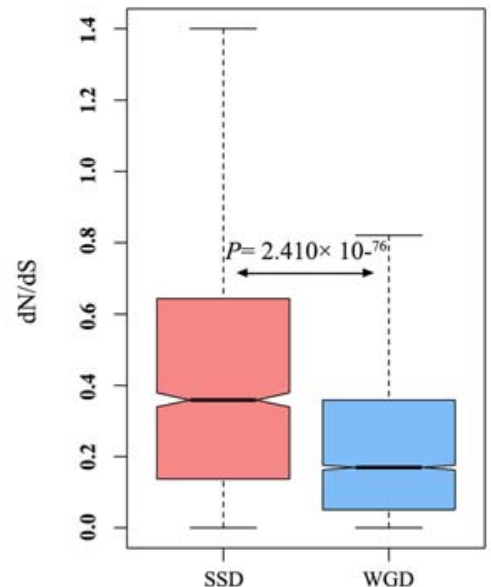

Using one-to-one Chimpanzee orthologs

Fig. 4 Differences in evolutionary rates of human small-scale and whole genome duplicates using Mouse (Mus musculus) and Chimpanzee (Pan troglodytes) one-to-one orthologs. Both the $d N(\mathbf{a})$ and the $\frac{d N}{d S}$ ratios (b) were used as the measurements of evolutionary rate. The SSDs are represented in brick red and WGDs are represented in blue. Exact P-values are provided in the figure and in Table 2

than the SSD pairs, irrespective of their sequence divergence for both the 'GO Biological Process' and 'GO Molecular Function' domains (Fig. 1, Table 1). We observed that these results are not influenced by duplicates having a large family size by conducting the same experiments using the closest duplicate pair only for both SSD and WGD duplicates (Additional file 1: Figure S1). We also observed that this difference is not due to the percentage identity based on which the SSD pairs are obtained, as using more stringent thresholds for determining SSD pairs also shows the similar trend (Additional file 1: Figure S2).
As the function of a protein is dependent on its localization in subcellular compartments [65], another possible mode of channelizing duplicated genes is in the subcellular localization of their encoded proteins [17]. Previous reports highlighted that the subcellular adaptation of duplicated proteins is also associated with the functional diversification [17, 47]. Consistent with this finding, we also observed a higher subcellular colocalization of the proteins encoded by SSD pairs (Table 1, Fig. 2; Additional file 1: Figure S3). This pattern is also opposite to that of yeast, where SSD pairs were more divergent in their subcellular localization, suggesting the human 

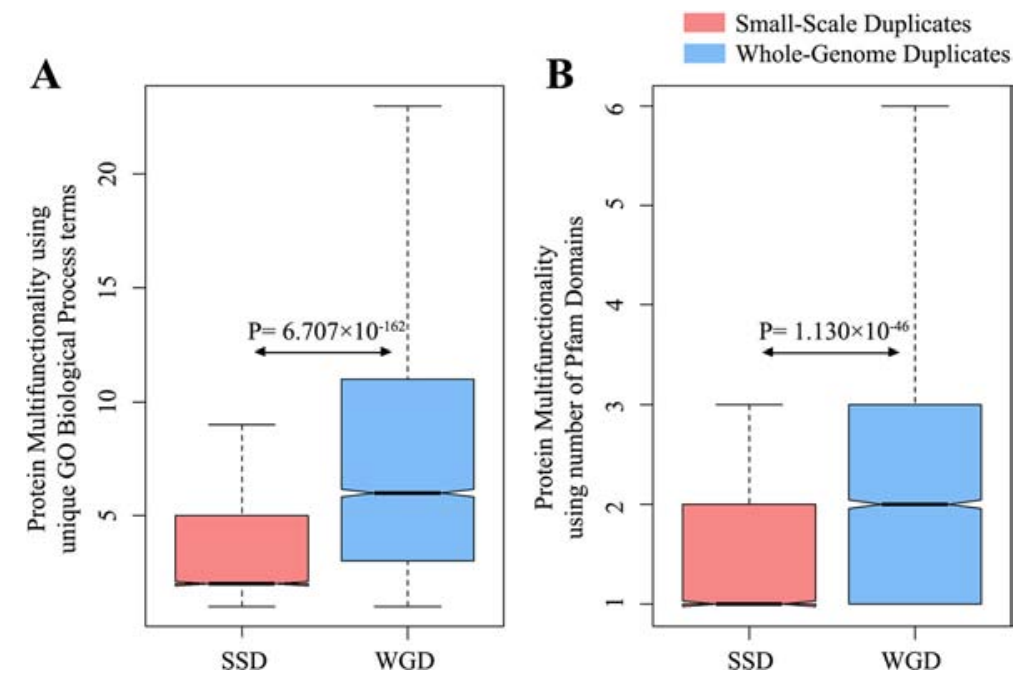

Fig. 5 Multifunctionality of human small-scale and whole-genome duplicates: a. Using their association with unique GO-Biological Processes. b. Using the number of Pfam domains. The SSDs are represented in brick red and WGDs are represented in blue. Exact P-values are provided in the figure

whole-genome duplicates have a higher probability of adapting themselves to new locations than the SSD counterparts. However, in higher eukaryotes having a tissue-level organization, gene duplication and the subsequent functional redundancy between the paralogs are often regulated by patterning their gene expression in different tissues [13-16, 66-68]. For example, the paralogs may express differentially in different tissues so that the amount of the produced protein remain at a steady level. Therefore, the spatial variation of gene expression can be treated as a possible candidate for the maintenance of duplicated pairs in humans. But the differences in gene expression patterns of SSD and WGD duplicated pairs were still not clear. Using high-throughput RNA-seq gene expression data of human for at least 27 normal tissues, we observed that the SSD pairs are more often coexpressed in the same tissue, whereas, WGD pairs tend to express differentially, i.e. in different tissues. This explains the idea that these WGD duplicates have not only adapted themselves to divergent functions or new locations, but also in divergent tissues. This also suggests a higher probability of specialization of expression patterns of human WGD pairs than the SSDs having the same level of sequence divergence (Fig. 3, Table 1). Using more stringent sequence identity for identification of SSDs also shows the similar trend (Additional file 1: Figure S4). Additionally, using closest paralogs to normalize the influence of duplicates with large gene families also shows that the differences between human SSD and WGD pairs hold true (Additional file 1: Figure S1). However, as humans are very distantly related with reference to the vertebrate whole- genome duplication event, we hypothesised that our results reflect the long-term evolutionary fates of genes originating from whole-genome duplication, with those originating from small-scale duplications. To prove our hypothesis, we firstly explored the influence of recent small-scale duplications in our dataset uding phylostratum rank as the age of SSD genes [69]. We differentiated the SSD pairs in two groups- youngSSD pairs and old-SSD pairs (see Additional file 2 for more details) and reperformed our overall analysis. We observed that the proportion of young SSDs are very low in our dataset $(Z=79.875$, confidence level $99 \%$; $P<1.00 \times 10^{-4}$, two sample Z-test) and differences between old-SSD and WGD genes still persist (Additional file 2: Figure S5). From another perspective, we used Xenopus tropicalis as a control and compare the attributes of small-scale and wholegenome duplicates in xenopus genome. Interestingly, both the SSD and WGD pairs shows high functional similarity in xenopus, with very little or no difference (Additional file 3: Figure S6). This also indicates that in course of vertebrate evolution, although initially both the SSD and WGD duplicates were similar in their attributes, the WGD genes were found to be more suitable candidates to diverge themselves to perform novel functions.

The higher functional divergence of human WGD genes and divergence in their subcellular and tissuespecific gene expression patterns lead us to investigate the differences in evolutionary conservation between SSD and WGD genes. In general, the duplicated genes tend to evolve faster than singletons just after duplication due to the increased functional redundancy, and 
subsequently upon its functional specialization, these duplicates evolve at a slower rate to maintain the functions to which it became adopted [43]. However, the human WGD genes are identified as the genes originated at the early vertebrate lineage, where the two rounds of genome duplication had happened. We observed a slower evolutionary rate in human WGD genes in contrast to SSD counterparts, which clearly demonstrates that the WGD genes has become adapted to new functions and lost its redundancy, and became slow evolving to maintain these functions (Fig. 4). The slower evolutionary rates and higher functional divergence of WGD genes indicate that the functions, to which they are adopted, are also evolutionarily conserved.

Our hypothesis that human WGDs have adapted to divergent functions and became evolutionarily conserved is further strengthened by the analysis of protein multifunctionality. The WGD genes and their encoded proteins tend to have higher multifunctionality than the SSD genes (Fig. 5), which strengthen our idea of higher adaptation of human WGD genes to new functions in contrast to SSD counterparts. However, besides the functional fate of duplicated genes, we were interested to comprehend the importance of such functions to the organism's life. Therefore, we also considered the importance of such functions to human. We used the gene essentiality along with the disease association as measurements of the vitality of a gene. Firstly we studied the effect of gene deletion to understand the functional restoration by the paralogous copy(ies). A recent study showed that the proportion of essential human gene is significantly higher in duplicates than in singletons [56]. Additionally, disease-associated genes were found to be enriched in duplicates [61]. Considering Mendelian disease genes, researchers also found WGDs to be more frequently disease-associated [39]. As our data contains two groups of duplicates which are quite different in their evolutionary genomic properties, we were curious to observe the proportion of essential genes and diseaseassociated genes (considering both Mendelian and Complex disease genes) among human SSD and WGD gene sets. We obtained a higher proportion of essential genes, as well as disease genes in the whole-genome duplicate set. Taken together, these results prove that the WGD genes have adapted themselves to serve more functions, which are more often crucial for humans, and may cause disease, sterility or even lethality upon disruption.

\section{Conclusions}

In summary, our results suggest that the human duplicates originated from WGD event in early vertebrate evolution are quite different from those originating spontaneously at a smaller-scale (SSD), but these differences are exactly opposite to that of yeast. The possible explanation for this scenario is that the human WGDs have been traced back from long time ago on the evolutionary scale, as in humans, we obtained the WGDs from two rounds of whole-genome duplication occurred in early vertebrate lineage. Additionally, the SSDs in our dataset are also enriched in ancient genes. Clearly, it suggests that both the human SSD and WGD genes have faced many evolutionary challenges than that in yeast. However, we found that in long evolutionary timespan, WGDs are more prone to diverge themselves in structure, function as well as expression to perform new and beneficial roles within the organism than the SSD genes. This also increases the chance to cause disease or lethality upon mutation on the WGD genes, due to the inability of their paralogous copies to restore the genedeletion fitness. However, why the ancient SSD and WGD genes show differences in their functional divergence, being evolutionarily similar in origin, is a matter of future investigation. In conclusion, our study provide an insight into the long-term evolutionary fate of duplicates originated from whole-genome duplication, rather than their immediate impact on the organism, to which the early studies with yeast $[20,41,44]$ were focussed.

\section{Methods \\ Identification of human small-scale and whole genome duplicates}

We obtained 22,447 human protein coding genes from the biomart interface of Ensembl version 77 [46] (http:// www.ensembl.org/biomart/martview). The whole-genome duplicate (WGD) pairs were obtained and compiled from two datasets: 1. Makino and McLysaght [39] and 2. OHNOLOGS (http://ohnologs.curie.fr/) [45]. We used the strict [q-score (outgroup) $<0.01$ and q-score] (self comparison) $<0.01$ ] dataset of OHNOLOGS database to discard false positives and maintain stringency of our data. All other duplicates were obtained from the biomart interface of Ensembl 77 [45] and termed as small-scale duplicates (SSD). We used $50 \%$ sequence identity with high paralogy confidence to assign paralogs, in order to retain old and/or distant paralogs. Finally, we obtained 34,746 duplicated pairs with 21,446 SSD and 13,300 WGD pairs comprising 4670 and 7070 genes, respectively (Additional file 4: Table S1).

As our dataset contains two groups of duplicates originated differentially in evolutionary time-scale, they are also different in terms of sequence divergence between duplicated pairs. The whole genome duplicates have originated during the evolution of early vertebrates and the small-scale duplicates have originated spontaneously at different times, thus, the latter may contain more recent duplicates with a possibility of being less diverged in sequence level. Therefore, it is necessary to remove 
the bias due to the differential sequence divergence of SSDs and WGDs for calculating their differences in their functional properties. For this, we binned our dataset according to the nonsynonymous nucleotide substitution per nonsynonymous sites $(\mathrm{dN})$ between each duplicated pairs, as the nonsynonymous substitutions bring change at protein level and older duplicate group (WGD) will tend to have higher $\mathrm{dN}$ than the newer one (SSD). Finally, both SSD and WGD duplicate pairs, we obtained five groups based on $\mathrm{dN}$ ranges between the paralogs - $\mathrm{dN}_{0.0-0.1}, \mathrm{dN}_{0.1-0.2}, \mathrm{dN}_{0.2-0.3}, \mathrm{dN}_{0.3-0.4}$ and $\mathrm{dN}>0.4$ and differentiated the evolutionary features between SSD and WGD genes in each dN range.

\section{Functional similarity}

The functions of human protein coding genes represented by their Gene Ontology terms were obtained from the biomart interface of Ensembl version 77 [46]. We considered the GO domains 'Biological process' as well as 'Molecular function' separately for functional similarity measurement. The functional similarity within each duplicate pair were calculated by their GO annotations using the following formula adapted from the Bayesian data integration method [44, 70]-

$$
\text { Functional Similarity }(i, j)=\frac{2 \times S(i, j)}{[\text { GO } \operatorname{terms}(i)+\mathbf{G O} \operatorname{terms}(\boldsymbol{j})]}
$$

Where 'i' and 'j' are duplicated pairs and 'S(i,j)' represents the Gene Ontology terms shared between the duplicated pairs 'i' and ' $j$ '.

\section{Subcellular localization}

The protein subcellular localization represented by the respective genes' Gene Ontology terms for the GO domain 'Cellular component' were obtained from the biomart interface of Ensembl version 77 [46]. Considering the Gene Ontology terms of a gene and its paralog, we obtained the subcellular compartment sharing for each SSD and WGD duplicate pairs. With the same formula used for functional similarity calculation mentioned previously, we calculated the subcellular compartment sharing for each duplicate pairs and compared the SSD and WGD genes of different $d N$ ranges (as mentioned above).

\section{Gene expression}

The RNA-seq gene expression data of human were taken from two databases- The gene expression values of 9113 duplicated genes in 27 different tissues (namely adipose tissue, adrenal gland, appendix, bone marrow, cerebral cortex, colon, duodenum, oesophagus, gallbladder, heart muscle, kidney, liver, lung, lymph node, ovary, pancreas, placenta, prostate, salivary gland, skin, small intestine, spleen, stomach, testis, thyroid gland, urinary bladder and uterus) were extracted from the human protein atlas Release 9 (http://www.proteinatlas.org/) $[49,71]$ and 9393 duplicate genes in 32 different tissues (namely adipose tissue, adrenal gland, ovary, appendix, bladder, bone marrow, cerebral cortex, colon, duodenum, endometrium, oesophagus, fallopian tube, gall bladder, heart, kidney, liver, lung, lymph node, pancreas, placenta, prostate, rectum, salivary gland, skeletal muscle, skin, small intestine, smooth muscle, spleen, stomach, testis, thyroid and tonsil) were obtained from Expression Atlas (http://www.ebi.ac.uk/gxa) [50, 72], which present stable repositories of experimental RNA-seq gene expression data in human tissues. The Pearson correlation coefficient (see formula below) was used to determine the expression profile similarity within the paralogous copies.

$$
=\frac{N \sum i j-\left(\sum i\right)\left(\sum j\right)}{\sqrt{\left[N \sum i^{2}-\left(\sum i\right)^{2}\right]\left[N \sum j^{2}-\left(\sum j\right)^{2}\right]}}
$$

Where ' $i$ ' and ' $j$ ' are paralogous pairs, ' $N$ ' is the total number of tissues, ' $\sum i j$ ' is the sum of the products of paired expression signal intensities, ' $\Sigma i$ ' sum of expression signal intensities for gene ' $i$ ', ' $\sum j$ ' is the sum of expression signal intensities for gene ' $j$ ', ' $\left(\sum i^{2}\right)^{\prime}$ ' is sum of squared expression signal intensities of gene ' $i$,' $\Sigma j^{2}$ ' is sum of squared expression signal intensities of gene $" j$.

\section{Evolutionary rate}

The oldest and widely used measurement of evolutionary rate calculates the evolutionary rate by using either $d N$ values [73], or the $\frac{d N}{d S}$ ratio $[74,75]$, where ' $\mathrm{dN}$ ' denotes Nonsynonymous nucleotide substitution per nonsynonymous sites and 'dS' stands for Synonymous nucleotide substitution per synonymous sites. For our study, we obtained one-to-one Mouse (Mus musculus) and Chimpanzee (Pan troglodytes) orthologs for each human genes to obtain the $\mathrm{dN}$ and dS values from the biomart interface of Ensembl version 77 [46]. Mutation saturation was controlled by discarding all $\mathrm{dS}$ values $\geq 3$ [76]. We discarded the genes having paralogous copies from both small-scale and whole-genome duplications and used the nonredundant set of 9386 genes with only SSD or only WGD paralogs, but not both. Considering these SSD-only and WGD-only pairs, we obtained two distinct sets of genes: 1. Genes (and its paralogous copies) involved in Smallscale duplication only (SSD only) (containing 3478 genes), and 2. Genes involved in Whole-genome duplication only (WGD only) (containing 5908 genes). The $\mathrm{dN}$ values and 
$\frac{d N}{d S}$ ratios between these groups were compared and used as the measurement of evolutionary rate.

\section{Multifunctionality}

The Multifunctionality of a gene and its encoded protein was measured by two approaches: A. Using their Gene Ontology annotation [77] for the GO domain 'biological process' from Ensembl Genome Browser [46], we calculated the unique biological processes of which a gene and its encoded protein(s) take part and used as the measurement of multifunctionality [51, 52], B. Additionally, we also considered the number of functional protein domains as proxy of Multifunctionality using Pfam protein families database. Finally, we compared the multifunctionality of SSD-only and WGD-only genes.

\section{Gene essentiality}

The human gene essentiality data were obtained from the Online GEne Essentiality (OGEE) database (http:// ogeedb.embl.de/\#overview:) [55]. After matching this essentiality data with our dataset, we finally obtained gene essentiality information of 2692 SSD-only and 5730 WGD-only genes. We compared the proportion of essential genes between these duplicate sets.

\section{Disease association}

Human disease genes were obtained from 'The Human Gene Mutation Database' (http://www.hgmd.cf.ac.uk/ac/ index.php) [63]. After discarding redundancy, we were able to identify 9668 disease genes of which, 9299 genes were matched to our dataset. This contains both the monogenic and the polygenic disease genes and is considered as human disease-associated genes. All other genes were termed 'non-disease genes' $(N=13148)$. We compared the proportion of disease genes among the SSD-only $(N=3478)$ and WGD-only $(N=5908)$ sets.

\section{Software}

We used the SPSS package (version 13) [78] and our in-house PERL-script for all statistical analyses. The $\mathrm{R}$ package [79] was used for data representation.

\section{Availability of supporting data}

The dataset of human small-scale and whole-genome duplicate pairs used in the study is available in Additional Table S1.

\section{Ethics statement}

The human data used in the study were collected from publicly available databases. Therefore ethics was not required for our study.

\section{Additional files}

Additional file 1: Figure S1. The differences between human small-scale and whole-genome duplicate pairs using the closest paralogs. Figure S2. Functional similarity between human small-scale duplicates with different sequence identity thresholds and whole-genome duplicate pairs. Figure S3. Subcellular co-localization between human small-scale and whole-genome duplicate pairs. Figure S4. Differences in gene expression correlation between human small-scale and whole-genome duplicate pairs. (PDF $662 \mathrm{~kb}$ )

Additional file 2: Contains Figure S5. The differences between human young small-scale duplicates (Young-SSD) and old small-scale duplicates (Old-SSD) with whole-genome duplicates. (PDF 209 kb)

Additional file 3: Contains Figure S6. The differences between Xenopus tropicalis small-scale and whole-genome duplicates. (PDF 379 kb)

Additional file 4: Contains Table S1. The human Small-Scale and Whole-Genome duplicate pairs used in the study. (XLSX 705 kb)

\section{Abbreviations}

PPI: protein-protein interaction network; SSD: small-scale duplicates; WGD: whole-genome duplicates; GO: Gene ontology; dN: Nonsynonymous nucleotide substitution per nonsynonymous sites; dS: Synonymous nucleotide substitution per synonymous sites.

\section{Competing interests}

The authors declare that they have no competing interests.

\section{Authors' contributions}

DA conceived, designed and performed the work. DA wrote the manuscript, TCG helped in planning of the work and drafting the manuscript. Both authors reviewed the manuscript.

\section{Acknowledgements}

We thank Dr. Sandip Chakrakraborty and Dr. Soumita Podder for technical advices. We also thank the Editor and two anonymous Reviewers for their constructive suggestions to improve our study. This work was supported by University Grants Commission (UGC) (Grant Sanction Letter No.F.2-8/2002

(SA-I) dated 04.10.2012 to D.A.).

Received: 28 September 2015 Accepted: 13 January 2016 Bt?

\section{References}

1. Ohno S, Wolf U, Atkin NB. Evolution from fish to mammals by gene duplication. Hereditas. 1968;59(1):169-87.

2. Ohno S. Evolution by Gene Duplication. New York: Springer-Verlag; 1970.

3. Stephens SG. Possible significances of duplication in evolution. Adv Genet. 1951:4:247-65.

4. Clark AG. Invasion and maintenance of a gene duplication. Proc Natl Acad Sci U S A. 1994;91(8):2950-4

5. Innan H, Kondrashov F. The evolution of gene duplications: classifying and distinguishing between models. Nat Rev Genet. 2010;11(2):97-108.

6. Teshima KM, Innan H. Neofunctionalization of duplicated genes under the pressure of gene conversion. Genetics. 2008;178(3):1385-98.

7. Taylor JS, Raes J. Duplication and divergence: the evolution of new genes and old ideas. Annu Rev Genet. 2004;38:615-43.

8. Kondrashov FA, Kondrashov AS. Role of selection in fixation of gene duplications. J Theor Biol. 2006;239(2):141-51.

9. Liang $\mathrm{H}$, Li W-H. Functional compensation by duplicated genes in mouse. Trends Genet. 2009;25(10):441-2.

10. Gu ZL, Steinmetz LM, Gu X, Scharfe C, Davis RW, Li WH. Role of duplicate genes in genetic robustness against null mutations. Nature. 2003;421(6918):63-6.

11. Li J, Musso G, Zhang Z. Preferential regulation of duplicated genes by microRNAs in mammals. Genome Biol. 2008;9(8):R132.

12. Chang AY-F, Liao B-Y. DNA methylation rebalances gene dosage after mammalian gene duplications. Mol Biol Evol. 2012;29(1):133-44. 
13. Qian W, Liao B-Y, Chang AY-F, Zhang J. Maintenance of duplicate genes and their functional redundancy by reduced expression. Trends Genet. 2010;26(10):425-30.

14. Ganko EW, Meyers BC, Vision TJ. Divergence in expression between duplicated genes in Arabidopsis. Mol Biol Evol. 2007;24(10):2298-309.

15. Li WH, Yang J, Gu X. Expression divergence between duplicate genes. Trends Genet. 2005:21(11):602-7.

16. Li Z, Zhang H, Ge S, Gu X, Gao G, Luo J. Expression pattern divergence of duplicated genes in rice. BMC Bioinformatics. 2009;10(Suppl 6):S8.

17. Marques AC, Vinckenbosh N, Brawand D, Kaessmann H. Functional diversification of duplicate genes through subcellular adaptation of encoded proteins. Genome Biol. 2008;9(3):R54.

18. Lynch M, Force A. The probability of duplicate gene preservation by subfunctionalization. Genetics. 2000;154(1):459-73.

19. Force A, Lynch M, Pickett FB, Amores A, Yan YL, Postlethwait J. Preservation of duplicate genes by complementary, degenerative mutations. Genetics. 1999:151(4):1531-45.

20. Hakes L, Pinney JW, Lovell SC, Oliver SG, Robertson DL. All duplicates are not equal: the difference between small-scale and genome duplication. Genome Biol. 2007:8(10):R209.

21. Adams KL, Wendel JF. Polyploidy and genome evolution in plants. Curr Opin Plant Biol. 2005;8(2):135-41.

22. Wendel JF. Genome evolution in polyploids. Plant Mol Biol. 2000;42(1):225-49.

23. Stebbins GL. Chromosomal Evolution in Higher Plants. New York: AddisonWesley; 1971.

24. Blanc G, Barakat A, Guyot R, Cooke R, Delseny I. Extensive duplication and reshuffling in the arabidopsis genome. Plant Cell. 2000;12(7):1093-101.

25. Kellis M, Birren BW, Lander ES. Proof and evolutionary analysis of ancient genome duplication in the yeast Saccharomyces cerevisiae. Nature. 2004; 428(6983):617-24

26. Wolfe $\mathrm{KH}$, Shields DC. Molecular evidence for an ancient duplication of the entire yeast genome. Nature. 1997;387(6634):708-13.

27. Dujon B, Sherman D, Fischer G, Durrens P, Casaregola S, Lafontaine I, et al. Genome evolution in yeasts. Nature. 2004;430(6995):35-44.

28. Brunet FG, Crollius HR, Paris M, Aury J-M, Gibert P, Jaillon O, et al. Gene loss and evolutionary rates following whole-genome duplication in teleost fishes. Mol Biol Evol. 2006;23(9):1808-16.

29. Zhou RJ, Cheng HH, Tiersch TR. Differential genome duplication and fish diversity. Rev Fish Biol Fisher. 2001;11(4):331-7.

30. Allendorf FW, Thorgaard GH. Tetraploidy and the evolution of salmonid fishes. In: Turner BJ, editor. Evolutionary Genetics of Fishes. New York: Plenum Press; 1984. p. 1-53.

31. Dehal $P$, Boore JL. Two rounds of whole genome duplication in the ancestral vertebrate. PLoS Biol. 2005;3(10):1700-8.

32. McLysaght A, Hokamp K, Wolfe KH. Extensive genomic duplication during early chordate evolution. Nat Genet. 2002;31(2):200-4.

33. Nakatani $Y$, Takeda H, Kohara Y, Morishita S. Reconstruction of the vertebrate ancestral genome reveals dynamic genome reorganization in early vertebrates. Genome Res. 2007:17(9):1254-65.

34. Chakraborty S, Ghosh TC. Evolutionary rate heterogeneity of core and attachment proteins in yeast protein complexes. Genome Biol Evol. 2013;5(7):1366-75

35. Papp B, Pal C, Hurst LD. Dosage sensitivity and the evolution of gene families in yeast. Nature. 2003:424(6945):194-7.

36. He XL, Zhang JZ. Higher duplicability of less important genes in yeast genomes. Mol Biol Evol. 2006;23(1):144-51.

37. Birchler JA, Veitia RA. The gene balance hypothesis: from classical genetics to modern genomics. Plant Cell. 2007;19(2):395-402.

38. Freeling M, Thomas BC. Gene-balanced duplications, like tetraploidy, provide predictable drive to increase morphological complexity. Genome Res. 2006;16(7):805-14.

39. Makino T, McLysaght A. Ohnologs in the human genome are dosage balanced and frequently associated with disease. Proc Natl Acad Sci U S A 2010;107(20):9270-4

40. Lynch M, Conery JS. The evolutionary fate and consequences of duplicate genes. Science. 2000;290(5494):1151-5.

41. Fares MA, Keane OM, Toft C, Carretero-Paulet L, Jones GW. The Roles of Whole-Genome and Small-Scale Duplications in the Functional Specialization of Saccharomyces cerevisiae Genes. PLoS Genet. 2013;9:e1003176.

42. Robinson-Rechavi M, Laudet V. Evolutionary rates of duplicate genes in fish and mammals. Mol Biol Evol. 2001;18(4):681-3.
43. Jordan IK, Wolf YI, Koonin EV. Duplicated genes evolve slower than singletons despite the initial rate increase. BMC Evol Biol. 2004;4:22.

44. Guan Y, Dunham MJ, Troyanskaya OG. Functional analysis of gene duplications in Saccharomyces cerevisiae. Genetics. 2007;175(2):933-43.

45. Singh PP, Arora J, Isambert $H$. Identification of ohnolog genes originating from whole genome duplication in early vertebrates, based on synteny comparison across multiple genomes. PLoS Comput Biol. 2015;11(7):e1004394.

46. Flicek P, Amode MR, Barrell D, Beal K, Billis K, Brent S, et al. Ensembl 2014. Nucleic Acids Res. 2014;42(D1):D749-55.

47. Byun-McKay SA, Geeta R. Protein subcellular relocalization: a new perspective on the origin of novel genes. Trends Ecol Evol. 2007; 22(7):338-44

48. Qian W, Zhang J. Protein subcellular relocalization in the evolution of yeast singleton and duplicate genes. Genome Biol Evol. 2009;1:198-204.

49. Uhlén M, Fagerberg L, Hallström BM, Lindskog C, Oksvold P, Mardinoglu A, et al. Tissue-based map of the human proteome. Science. 2015;347(6220): 1260419.

50. Petryszak R, Burdett T, Fiorelli B, Fonseca NA, Gonzalez-Porta M, Hastings E, et al. Expression Atlas update- a database of gene and transcript expression from microarray- and sequencing-based functional genomics experiments. Nucleic Acids Res. 2014;42(D1):D926-32.

51. Satake M, Kawata M, McLysaght A, Makino T. Evolution of vertebrate tissues driven by differential modes of gene duplication. DNA Res. 2012;19:305-16.

52. Podder S, Mukhopadhyay P, Ghosh TC. Multifunctionality dominantly determines the rate of human housekeeping and tissue specific interacting protein evolution. Gene. 2009;439(1-2):11-6.

53. Salathe M, Ackermann M, Bonhoeffer $\mathrm{S}$. The effect of multifunctionality on the rate of evolution in yeast. Mol Biol Evol. 2006;23(4):721-2.

54. Finn RD, Bateman A, Clements J, Coggill P, Eberhardt RY, Eddy SR, et al Pfam: the protein families database. Nucleic Acids Res. 2014; 42(Database):D222-30.

55. Chen W-H, Minguez P, Lercher MJ, Bork P. OGEE: an online gene essentiality database. Nucleic Acids Res. 2012:40(D1):D901-6.

56. Acharya D, Mukherjee D, Podder S, Ghosh TC. Investigating different duplication pattern of essential genes in mouse and human. PLoS One. 2015;10(3):e0120784-4.

57. Hsiao T-L, Vitkup D. Role of duplicate genes in robustness against deleterious human mutations. PLoS Genet. 2008;4(3):e1000014-4.

58. Dean EJ, Davis JC, Davis RW, Petrov DA. Pervasive and Persistent Redundancy among Duplicated Genes in Yeast. Plos Genet. 2008;4(7): e1000113.

59. Wagner A. Gene duplications, robustness and evolutionary innovations. Bioessays. 2008;30(4):367-73.

60. Forslund K, Schreiber F, Thanintorn N, Sonnhammer ELL. OrthoDisease: tracking disease gene orthologs across 100 species. Brief Bioinform. 2011;12(5):463-73.

61. Dickerson JE, Robertson DL. On the origins of mendelian disease genes in man: the impact of gene duplication. Mol Biol Evol. 2012;29(1):61-9.

62. Chen WH, Zhao XM, van Noort V, Bork P. Human monogenic disease genes have frequently functionally redundant paralogs. PLoS Comput Biol. 2013;9(5):e1003073.

63. Stenson PD, Ball EV, Mort M, Phillips AD, Shaw K, Cooper DN. The Human Gene Mutation Database (HGMD) and its exploitation in the fields of personalized genomics and molecular evolution. Curr Protoc Bioinform. 2012;Chapter 1:Unit1.13-Unit11.13.

64. Zhang JZ. Evolution by gene duplication: an update. Trends Ecol Evol. 2003;18(6):292-8.

65. Emanuelsson $\mathrm{O}$, von Heijne $\mathrm{G}$. Prediction of organellar targeting signals. BBA-Mol Cell Res. 2001;1541(1-2):114-9.

66. Chen K, Zhang Y, Tang T, Shi S. Cis-regulatory change and expression divergence between duplicate genes formed by genome duplication of Arabidopsis thaliana. Chinese Sci Bull. 2010;55(22):2359-65.

67. Ha M, Kim E-D, Chen ZJ. Duplicate genes increase expression diversity in closely related species and allopolyploids. Proc Natl Acad Sci U S A. 2009; 106(7):2295-300

68. Leach LJ, Zhang Z, Lu C, Kearsey MJ, Luo Z. The role of Cis-Regulatory motifs and genetical control of expression in the divergence of yeast duplicate genes. Mol Biol Evol. 2007;24(11):2556-65.

69. Neme R, Tautz D. Phylogenetic patterns of emergence of new genes support a model of frequent de novo evolution. BMC Genomics. 2013;14(1):117. 
70. Podder S, Ghosh TC. Insights into the molecular correlates modulating functional compensation between monogenic and polygenic disease gene duplicates in human. Genomics. 2011;97(4):200-4.

71. Uhlen M, Bjorling E, Agaton C, Szigyarto CA, Amini B, Andersen E, et al. A human protein atlas for normal and cancer tissues based on antibody proteomics. Mol Cell Proteomics. 2005;4(12):1920-32.

72. Kapushesky M, Adamusiak T, Burdett T, Culhane A, Farne A, Filippov A, et al. Gene Expression Atlas update-a value-added database of microarray and sequencing-based functional genomics experiments. Nucleic Acids Res. 2012;40(D1):D1077-81.

73. Begum T, Ghosh TC. Understanding the effect of secondary structures and aggregation on human protein folding class evolution. J Mol Evol. 2010;71(1):60-9.

74. Wall DP, Hirsh AE, Fraser HB, Kumm J, Giaever G, Eisen MB, et al. Functional genomic analysis of the rates of protein evolution. Proc Natl Acad Sci U S A. 2005;102(15):5483-8.

75. Chen FC, Liao BY, Pan CL, Lin HY, Chang AY. Assessing determinants of exonic evolutionary rates in mammals. Mol Biol Evol. 2012;29(10):3121-9.

76. Begum T, Ghosh TC. Elucidating the genotype-phenotype relationships and network perturbations of human shared \& specific disease genes from an evolutionary perspective. Genome Biol Evol. 2014;6(10):2741-53.

77. Gene Ontology Consortium. The Gene Ontology (GO) database and informatics resource. Nucleic Acids Res. 2004;32 suppl 1:D258-61.

78. Nie N, Bent D, Hull C. SPSS: statistical package for the social sciences. New York: McGraw-Hill; 1970.

79. Ihaka R, Gentleman R. R: a language for data analysis and graphics. J Comput Graph Stat. 1996;5:299-314.

\section{Submit your next manuscript to BioMed Central and we will help you at every step:}

- We accept pre-submission inquiries

- Our selector tool helps you to find the most relevant journal

- We provide round the clock customer support

- Convenient online submission

- Thorough peer review

- Inclusion in PubMed and all major indexing services

- Maximum visibility for your research

Submit your manuscript at www.biomedcentral.com/submit 